\title{
Vocal Fold Hyperplastic Lesions: an Evaluation of Surgical Outcome with Videolaryngostroboscopy
}

\author{
Melek Kezban Gürbüz1', Leman Birdane², Mustafa Fuat Açıkalın³ , Ertuğrul Çolak, Erkan Özüdoğru', Cemal Cingi', \\ Armağan İncesulu ${ }^{1}$
}

${ }^{1}$ Department of Otorhinolaryngology, Eskişehir Osmangazi University Faculty of Medicine, Eskişehir, Turkey

${ }^{2}$ Clinic of Otorhinolaryngology, Eskişehir Yunus Emre State Hospital, Eskişehir, Turkey

${ }^{3}$ Department of Pathology, Eskişehir Osmangazi University Faculty of Medicine, Eskişehir, Turkey

${ }^{4}$ Department of Biostatistics, Eskişehir Osmangazi University Faculty of Medicine, Eskişehir, Turkey

\begin{abstract}
Background: Vocal fold hyperplastic lesions are premalignant lesions that can be treated effectively by removal of the lesions surgically.

Aims: The aim of this study was to discuss the success of surgery in patients with vocal fold hyperplastic lesions in terms of preserving vibratory function by comparing the preoperative and postoperative videolaryngostroboscopy findings.

Study Design: The medical charts and videolaryngostroboscopic recordings of patients diagnosed with hyperplastic lesions on the vocal folds were reviewed retrospectively.

Methods: Twenty seven patients with unilateral lesions who underwent type1 subepithelial cordectomy were enrolled in the study. The videolaryngostroboscopic recordings were evaluated by three raters who were not the operating surgeon and who were blinded to the histology of patients. To evaluate the videolaryngostroboscopic findings, a form, which is a modification of criteria described by Hirano and Bless, was used. Preoperative and 6th month postoperative videolaryngostroboscopic recordings were compared with each other and with recordings of the control group, which included 50 healthy volunteers.
\end{abstract}

Results: All videolaryngostroboscopic findings, except false cord vibration, were significantly improved after surgery.

Conclusion: The principle of vocal fold surgery in patients with benign lesions is to preserve the vibratory tissue. This principle also applies to patients with hyperplastic lesions that are premalignant. The hydrodissection technique may be beneficial for this purpose.

Key Words: Vocal fold, hyperplastic lesion, vibratory tissue, surgery, videolaryngostroboscopy

Received: 02.09.2012

Accepted: 07.10.2012

\section{Introduction}

Hyperplastic lesions were classified according to the World Health Organisation (WHO) criteria into five groups: squamous hyperplasia, mild dysplasia, moderate dysplasia, severe dysplasia and carcinoma in situ (1-3). These premalignant lesions often appear as thickened whitish, greyish or reddish mucosa and tend to be located on the vibratory mucosa of the vocal fold $(4,5)$. They are more frequent in patients who smoke, use their voice improperly or suffer from reflux, and they lead to cause deterioration in voice quality. If they are detected at an early stage, they might be kept under control with surgical treatment before transformation into invasive carcinoma. For detection of the lesion, indirect laryngoscopy, flexible endoscopy and videolaryngostroboscopy (VLS) can be used. The benefit of VLS is that it can depict the vibratory function of the vocal folds.

Removal of the lesion with elimination of the risk factors is the most effective treatment for vocal fold hyperplastic le- sions. Simple excision or type 1 endoscopic subepithelial cordectomy, as suggested by the European Laryngeal Society, is a widely used technique in current surgical practice (6). It is important to do minimum damage to the vibratory tissues of the vocal fold, regardless of which technique is used, especially in patients who use their voice professionally.

There are many studies in the literature dealing with the aetiology, prevalence and treatment results of vocal fold hyperplastic lesions, but studies evaluating the effects of surgical treatment on the vocal fold vibratory function are rare. In this study we aimed to discuss the success of surgery in patients with vocal fold hyperplastic lesions in terms of preserving vibratory function by comparing the preoperative and postoperative VLS findings.

\section{Material and Methods}

The ethical approval was obtained for the study (reference number: 2009-28). 


\section{Study Design}

The medical charts of patients diagnosed with hyperplastic lesions on vocal folds between January 2002 and January 2011 were retrospectively reviewed. The inclusion criteria for patients were as follows: a unilateral lesion on vocal folds, operated upon with type 1 subepithelial cordectomy using the hydrodissection method and had regular follow-up for at least 1 year. Patients having undergone previous laryngeal surgery and those who were re-operated upon because of recurrence within 6 months were excluded from the study. Twenty-seven patients met the inclusion criteria and were enrolled in the study. A data collection form was used to record information on demographic characteristics (age, history of smoking, professional), results of biopsy and follow-up (follow-up time, transformation into invasive carcinoma).

Preoperative and $6^{\text {th }}$ month postoperative VLS recordings of 27 patients were documented.

The control group of 50 healthy volunteers was created and underwent VLS.

\section{Assessment of VLS parameters}

The VLS unit consisted of a Karl Storz 8020 stroboscopic light source, Sony monitor, Telecam-C camera and its control unit, neck microphone, MAl Condenser voice recording microphone, and a Hopkins 8707 DJ-90 degree Karl Storz telescope. Acquired digital records were archived by Scope View Tigers Inc Seattle USA computerized soft. The evaluation of VLS recordings of patients and the control group was performed by three raters who were not the operating surgeon and who were blinded to the histology of patients. A form, which is a modification of the criteria described by Hirano and Bless, was used for evaluation (7). The reliability of this form is statistically confirmed by Cronbach's Alpha analysis (Cronbach's Alpha is 0.61 for control, 0.848 for preoperative and 0.847 for postoperative). The evaluated parameters are shown in Table 1.

The percent of vocal fold edge irregularity was calculated by the proportion of the length of the irregular portion to that of entire vocal fold on paused images obtained by Scope View software. The evaluation of latero-medial movement amplitude, mucosal wave, the non-vibratory portion of the vocal fold, periodicity and compression of the false cords was performed according to the criteria described by Hirano and Bless (7).

The percent of supraglottic antero-posterior contraction was also calculated by measuring the proportion of the distance between the epiglottic petiolus and the arythenoids on the paused images during inspiration and phonation.

\section{Statistical analysis}

The ages of groups were compared using Student's ttest. Spearman correlation analysis was used to determine the relation between age and VLS findings. Values for the control group were compared with the patient preoperative and postoperative ( $6^{\text {th }}$ month) values of VLS findings by using the Mann-Whitney $U$ test. In the patient group, preoperative and postoperative values of VLS findings were compared by using Wilcoxon Signed Rank test. The Intraclass Correlation
Table 1. Correlation between age and VLS recordings of patients (Spearman's rank correlation test)

\begin{tabular}{|c|c|c|c|}
\hline \multirow[b]{2}{*}{ VLS PARAMETERS } & \multicolumn{3}{|c|}{ AGE } \\
\hline & R1 & $\mathbf{R 2}$ & R3 \\
\hline \multirow{4}{*}{$\begin{array}{l}\text { Percent of vocal } \\
\text { fold edge } \\
\text { irregularity }\end{array}$} & $r^{1}=0.102$ & $r^{1}=0.102$ & $r^{1}=0.043$ \\
\hline & $\mathrm{p}^{1}=0.612$ & $\mathrm{p}^{1}=0.612$ & $p^{1}=0.833$ \\
\hline & $r^{2}=0.188$ & $r^{2=0}=188$ & $r^{2}=0.190$ \\
\hline & $p^{2}=0.349$ & $\mathrm{p}^{2}=0.349$ & $p^{2}=0.344$ \\
\hline \multirow{4}{*}{$\begin{array}{l}\text { LM Movement } \\
\text { Amplitude }\end{array}$} & $r^{1}=-0.001$ & $r^{1}=0.060$ & $r^{1}=-0.001$ \\
\hline & $\mathrm{p}^{1}=0.995$ & $\mathrm{p}^{1}=0.765$ & $\mathrm{p}^{2}=0.319$ \\
\hline & $p^{2}=0.364$ & $p^{1}=0.995$ & $r^{2}=0.133$ \\
\hline & $r^{2}=0.199$ & $r^{2}=0.182$ & $\mathrm{p}^{2}=0.508$ \\
\hline \multirow[t]{4}{*}{ Mucosal Wave } & $r^{1}=0.033$ & $r^{1}=0.013$ & $r^{1}=-0.034$ \\
\hline & $p^{1}=0.869$ & $\mathrm{p}^{1}=0.947$ & $p^{1}=0.867$ \\
\hline & $r^{2}=0.009$ & $r^{2}=-0.041$ & $r^{2}=-0.033$ \\
\hline & $\mathrm{p}^{2}=0.965$ & $p^{2}=0.839$ & $\mathrm{p}^{2}=0.872$ \\
\hline \multirow{4}{*}{$\begin{array}{l}\text { Non-vibratory } \\
\text { portion of } \\
\text { vocal fold }\end{array}$} & $r^{1}=-0.132$ & $r^{1}=0.333$ & $r^{1}=-0.104$ \\
\hline & $\mathrm{p}^{1} .=0.510$ & $\mathrm{p}^{1}=0.090$ & $p^{1}=0.604$ \\
\hline & $r^{2}=0.129$ & $r^{2}=0.242$ & $r^{2}=0.238$ \\
\hline & $\mathrm{p}^{2}=0.521$ & $p^{2}=0.224$ & $\mathrm{p}^{2}=0.232$ \\
\hline \multirow[t]{4}{*}{ Periodicity } & $r^{1}=0.333$ & $r^{1}=0.329$ & $r^{1}=0.244$ \\
\hline & $\mathrm{p}^{1}=0.090$ & $p^{1}=0.093$ & $\mathrm{p}^{1}=0.221$ \\
\hline & $r^{2}=-0.003$ & $r^{2}=-0.055$ & $r^{2}=-0.094$ \\
\hline & $\mathrm{p}^{2}=0.987$ & $\mathrm{p}^{2}=0.783$ & $p^{2}=0.642$ \\
\hline \multirow{4}{*}{$\begin{array}{l}\text { Compression of } \\
\text { false cord }\end{array}$} & $r^{1}=0.329$ & $r^{1}=-0.006$ & $r^{1}=0.316$ \\
\hline & $p^{1}=0.093$ & $p^{1}=0.978$ & $p^{1}=0.108$ \\
\hline & $r^{2}=0.154$ & $r^{2}=0.183$ & $r^{2}=0.183$ \\
\hline & $p^{2}=0.443$ & $\mathrm{p}^{2}=0.360$ & $\mathrm{p}^{2}=0.360$ \\
\hline \multirow{4}{*}{$\begin{array}{l}\text { False cord } \\
\text { vibration }\end{array}$} & $r^{1}=0.067$ & $\mathrm{p}^{2}=0.694$ & $r^{1}=0.065$ \\
\hline & $\mathrm{p}^{1}=0.740$ & $\mathrm{p}^{1}=0.371$ & $\mathrm{p}^{1}=0.749$ \\
\hline & $r^{2}=0.079$ & $r^{2}=0.152$ & $r^{2}=0.079$ \\
\hline & $r^{1}=0.179$ & $p^{2}=0.448$ & $p^{2}=0.694$ \\
\hline \multirow{4}{*}{$\begin{array}{l}\text { Percent of } \\
\text { supraglottic antero- } \\
\text { posterior contraction }\end{array}$} & $r^{1}=0.179$ & $r^{1}=0.188$ & $r^{1}=0.145$ \\
\hline & $\mathrm{p}^{1}=0.371$ & $\mathrm{p}^{1}=0.349$ & $\mathrm{p}^{1}=0.472$ \\
\hline & $r^{2}=-0.079$ & $r^{2}=-0.079$ & $r^{2}=-0.030$ \\
\hline & $p^{2}=0.696$ & $\mathrm{p}^{2}=0.696$ & $p^{2}=0.883$ \\
\hline \multicolumn{4}{|c|}{$\begin{array}{l}\text { R1: rater } 1 ; \text { R2: rater } 2 \text {; R3: rater } 3 \\
r^{1}: \text { preoperative correlation coefficient; } p^{1}=\text { preoperative } p \text { value } \\
r^{2} \text { : postoperative correlation coefficient; } p^{2}=\text { postoperative } p \text { value }\end{array}$} \\
\hline
\end{tabular}


Coefficient (ICC) was also used to assess inter-rater reliability. Analyses were performed with SPSS 15.0 for Windows and MedCalc 11.1 for Windows. VLS findings that were measured as scores were expressed as mean, standard error of mean (SE), median and $25^{\text {th }}-75^{\text {th }}$ percentiles (PCTL). $p<0.05$ was accepted as statistically significant.

\section{Results}

\section{Characteristics of patient and control group}

The patient group consisted of 27 males with a mean age of 60.44 (39-81) years. The control group consisted of 20 (40\%) females, 30 (60\%) males and the mean age was 46.14 (27-69) years. A statistically significant difference was found $(p<0.001)$ between the patient group and the control group in respect of age. However, this difference had no effect on the VLS findings. The Spearman correlation coefficients were found not to be significant, although there was a significant difference between the control and patient groups according to age. None of the patients and none of the subjects of the control group used their voice professionally. All of the patients and 8 (16\%) subjects of the control group were smokers. All patients had a unilateral lesion, but none of the subjects of the control group had any laryngeal complaint or pathology.

\section{Technique of surgery}

All of the patients underwent type 1 subepithelial cordectomy. All operations were done under general anaesthesia with an operating microscope using the hydrodissection method. In this method, saline was injected into Reinke's space at the onset of the surgical procedure (Figure 1).

\section{Findings of histopathological examinations}

One (3.7\%) patient had squamous hyperplasia, 8 (29.6\%) patients had mild, 6 (22.2\%) patients had moderate, 5 (18.5\%) patients had severe dysplasia and 7 (25.9\%) patients had carcinoma in situ at the first biopsy.

\section{Findings of VLS}

The comparison of VLS parameters evaluated by three raters is reported in Table 2 . The results show that we had a statistically and significantly improvement in all parameters except false cord vibration after surgery. ICCs for inter-rater

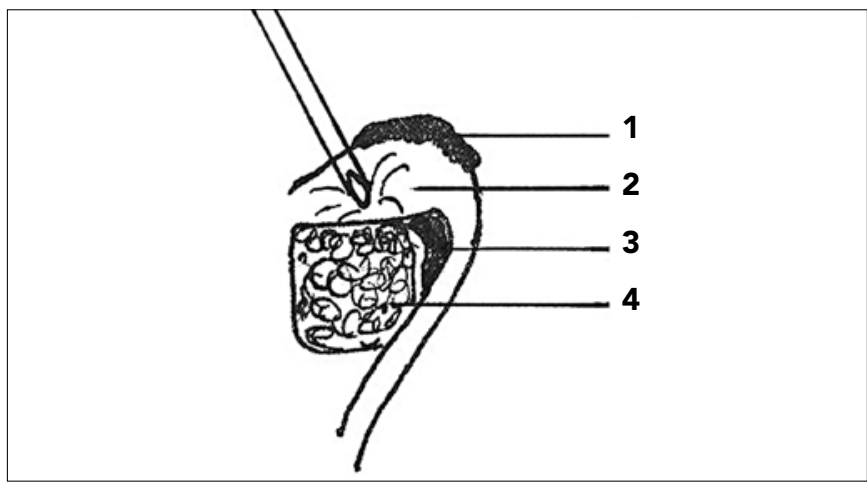

Figure 1. Hyperplastic lesion (1), Reinke's space (2), Vocal ligament (3), Vocalis muscle (4) reliability are shown in Table 3. Most of the ICCs indicated that the measurements of the three raters were reliable (ICC $>0.85)$.

\section{Follow-up}

The mean follow-up time was 48.1 (13-108) months. Ten (37.0\%) patients underwent repeated biopsies because of recurrence. One of 10 had squamous hyperplasia, another had severe dysplasia, and the remaining 8 had carcinoma in situ at first diagnosis. The mean number of repeat biopsies was 1.3 (1-3). Invasive carcinoma was detected in $3(11.1 \%)$ patients by repeated biopsies.

\section{Discussion}

Vocal fold hyperplastic lesions are precancerous lesions that demonstrate a loss of cellular maturation and cytological atypia (8). They are more frequent in men and in smokers. Similarly, the majority of our patients were male and all were smokers.

Table 2. Correlation between age and VLS recordings of control group (Spearman's rank correlation test)

\begin{tabular}{|c|c|c|c|}
\hline \multirow[b]{2}{*}{ VLS PARAMETERS } & \multicolumn{3}{|c|}{ AGE } \\
\hline & R1 & R2 & R3 \\
\hline Percent of vocal & $r=*$ & $r=*$ & $r=*$ \\
\hline fold edge irregularity & $p=*$ & $p={ }^{*}$ & $p={ }^{*}$ \\
\hline LM Movement & $r=0.026$ & $r=0.026$ & $r=0.071$ \\
\hline Amplitude & $p=0.860$ & $p=0.860$ & $p=0.626$ \\
\hline \multirow[t]{2}{*}{ Mucosal Wave } & $r=-0.186$ & $r=-0.186$ & $r=-0.251$ \\
\hline & $p=0.195$ & $p=0.195$ & $p=0.079$ \\
\hline Non-vibratory & $r=*$ & $r=*$ & $r=*$ \\
\hline portion of vocal fold & $p={ }^{*}$ & $p=*$ & $p={ }^{\star}$ \\
\hline \multirow[t]{2}{*}{ Periodicity } & $r=0.216$ & $r=0.216$ & $r=0.225$ \\
\hline & $p=0.132$ & $p=0.132$ & $p=0.117$ \\
\hline Compression & $r=0.054$ & $r=0.054$ & $r=0.042$ \\
\hline of false cord & $p=0.710$ & $p=0.710$ & $p=0.773$ \\
\hline False cord & $r=0.022$ & $r=0.022$ & $r=0.022$ \\
\hline vibration & $p=0.880$ & $p=0.880$ & $p=0.880$ \\
\hline Percent of & $r=0.135$ & $r=0.135$ & $r=0.046$ \\
\hline $\begin{array}{l}\text { supraglottic antero- } \\
\text { posterior contraction }\end{array}$ & $p=0.349$ & $p=0.349$ & $p=0.750$ \\
\hline \multicolumn{4}{|c|}{$\begin{array}{l}\text { R1: rater 1; R2: rater } 2 ; R 3: \text { rater } 3 \\
r^{1}: \text { preoperative correlation coefficient; } p^{1}=\text { preoperative } p \text { value } \\
r^{2}: \text { postoperative correlation coefficient; } p^{2}=\text { postoperative } p \text { value }\end{array}$} \\
\hline
\end{tabular}


Table 3. Comparison of VLS recordings of control group with gender of control group (Mann-Whitney U test)

\begin{tabular}{|c|c|c|c|}
\hline & $\begin{array}{c}\text { Male } \\
\text { Mean士standard error } \\
\text { Median }\left(25^{\text {th }}-75^{\text {th }}\right) \%\end{array}$ & $\begin{array}{c}\text { Female } \\
\text { Mean士standard error } \\
\text { Median }\left(25^{\text {th }}-75^{\text {th }}\right) \%\end{array}$ & $\mathbf{p}$ \\
\hline \multirow[t]{3}{*}{ Percent of vocal fold edge irregularity } & R1: $0 \pm 0$ & R1: $0 \pm 0$ & R1: 1.000 \\
\hline & R2: $0 \pm 0$ & $\mathrm{R} 2: 0 \pm 0$ & R2: 1.000 \\
\hline & R3: $0 \pm 0$ & R3: $0 \pm 0$ & R3: 1.000 \\
\hline \multirow[t]{3}{*}{ LM Movement Amplitude } & $\mathrm{R} 1: 0.31 \pm 0.10$ & $R 1: 0.19 \pm 0.08$ & R1: 0.458 \\
\hline & R2: $0.31 \pm 0.10$ & R2: $0.19 \pm 0.08$ & R2: 0.458 \\
\hline & $\mathrm{R} 3: 0.34 \pm 0.10$ & R3: $0.19 \pm 0.08$ & R3:0.321 \\
\hline \multirow[t]{3}{*}{ Mucosal Wave } & $\mathrm{R} 1: 0.52 \pm 0.10$ & $\mathrm{R} 1: 0.38 \pm 0.10$ & R1: 0.427 \\
\hline & $\mathrm{R} 2: 0.52 \pm 0.10$ & R2: $0.38 \pm 0.10$ & R2: 0.427 \\
\hline & R3: $0.52 \pm 0.10$ & R3: $0.43 \pm 0.11$ & R3: 0.635 \\
\hline \multirow[t]{3}{*}{ Non-vibratory portion of vocal fold } & $\mathrm{R} 1: 0 \pm 0$ & $\mathrm{R} 1: 0 \pm 0$ & R1: 1.000 \\
\hline & $\mathrm{R} 2: 0 \pm 0$ & $\mathrm{R} 2: 0 \pm 0$ & R2: 1.000 \\
\hline & R3: $0 \pm 0$ & R3: $0 \pm 0$ & R3: 1.000 \\
\hline \multirow[t]{3}{*}{ Periodicity } & $R 1: 0.38 \pm 0.09$ & $\mathrm{R} 1: 0.38 \pm 0.10$ & R1: 0.991 \\
\hline & R2: $0.38 \pm 0.09$ & R2: $0.38 \pm 0.10$ & R2: 0.991 \\
\hline & R3: $0.38 \pm 0.09$ & R3: $0.48 \pm 0.11$ & R3: 0.498 \\
\hline \multirow[t]{3}{*}{ Compression of false cord } & $R 1: 0.38 \pm 0.09$ & $\mathrm{R} 1: 0.24 \pm 0.11$ & R1: 0.200 \\
\hline & R2: $0.38 \pm 0.09$ & $\mathrm{R} 2: 0.24 \pm 0.11$ & R2: 0.200 \\
\hline & R3: $0.38 \pm 0.09$ & R3: $0.29 \pm 0.14$ & R3: 0.253 \\
\hline \multirow[t]{3}{*}{ False cord vibration } & $\mathrm{R} 1: 0.14 \pm 0.08$ & $\mathrm{R} 1: 0 \pm 0$ & R1: 0.133 \\
\hline & R2: $0.14 \pm 0.08$ & R2: $0 \pm 0$ & R2: 0.133 \\
\hline & R3: $0.14 \pm 0.08$ & R3: $0 \pm 0$ & R3: 0.133 \\
\hline Percent of supraglottic & $R 1: 1.00 \pm 0.12$ & $\mathrm{R} 1: 1.05 \pm 0.14$ & R1: 0.799 \\
\hline \multirow[t]{2}{*}{ antero-posterior contraction } & R2: $1.00 \pm 0.12$ & R2: $1.05 \pm 0.14$ & R2: 0.799 \\
\hline & R3: $1.07 \pm 0.13$ & R3: $1.10 \pm 0.15$ & R3: 0.897 \\
\hline
\end{tabular}

The ratio of transformation into invasive carcinoma of hyperplastic lesions was reported as about $10 \%$ in the literature (9). In the present study, invasive carcinoma was detected in $3(11.1 \%)$ patients; recurrence was detected in $10(37.0 \%)$ patients. Therefore, frequent and careful follow-up is necessary, especially in cases with recurrent lesions. It is obvious that assessment of recurrence or disease progression allows the early diagnosis of malignancy and the early detection of cancer is vital to improve cancer survival rates. For this reason, an objective documentation of laryngeal images with laryngeal rigid telescopic or flexible endoscopic examination is recommended during the follow-up of patients with vocal fold hyperplastic lesions, as the images obtained can be compared with the findings of former examinations at any time. We always prefer VLS for the follow-up of patients with premalignant lesions, because VLS can also allow the evaluation of the effects of surgical treatment on the vocal fold vibratory function. Voice recordings or some questionnaires can also be used to evaluate the success of surgery of patients undergoing voice surgery. We also use all of these examinations in our clinical practice, but in the present study, we did not include the evaluation of the voice recordings and questionnaires in the present study. This is because we frequently obtain variable results in objective voice analysis and answers of questionnaires in the patients with vocal fold hyperplastic lesions, even in short intervals. We believe that 
this inconsistency may be due to unstable psychological status, such as anxiety for possible malignant transformation of the disease and excessive expectation for the treatment of patients.

The main causes of hoarseness or bad voice quality in patients with vocal fold hyperplastic lesions are the irregularity of the vocal fold free edge(s) and the presence of any non-vibrating portions of the vocal fold(s). The irregularity prevents complete glottic closure during phonation and diminishes vibration. Therefore, it is desired to reconstitute the smooth re-epithelialisation on the vocal fold's free edge after excision of the lesion. In the present study, we achieved significant improvement of free edge irregularities postoperatively.

The reason for non-vibrating portions of vocal fold(s) in patients with hyperplastic lesions is mostly a regional mass increase on the vocal fold. However non-vibrating portions can also occur as a postoperative complication due to scarring at the surgical site. Therefore, maximum care should be taken in order to minimise any harm to vocal ligament during excision. Otherwise, any fibrosis developing in the region might lead to scarring and the persistence of a nonvibratory portion. In order to prevent this complication, safe excision can be performed with the help of the hydrodissection method (Figure 1). The present study demonstrated that non-vibratory portions were significantly decreased postoperatively. This result indicates that the preoperative non-vibratory segments were due to the mass effect of the lesion.

The main parameters used for evaluating vocal fold vibratory function with VLS are mucosal wave and latero-medial movement amplitude of vocal fold edges. Increased mass (polyp, tumour, premalignant lesions) on the vocal fold leads to a decrease in latero-medial movement amplitude and mucosal wave; therefore, the voice gets worse (7). However, decreased latero-medial movement and a decrease in the mucosal wave can occur as a result of an excessive resection of the superficial layer of the lamina propria in surgery leading to adherence of the epithelium to the vocal ligament (10). In the present study, we detected a significant improvement of mucosal wave and latero-medial movement amplitude parameters after surgery. This improvement might indicate that a meticulous surgical technique helps to protect the vocal fold functions by minimising the loss of the lamina propria and scars at the surgical site.

One of the important parameters associated with vocal performance is periodicity. This refers to the regularity of successive vocal vibratory cycles. Normal vibratory activity is regular and periodic. Glottic lesions or lung diseases blocking the regular delivery of the expiration air to the glottis can prevent periodic vibration (7). Vocal fold hyperplastic lesions may also affect the periodicity. Similarly, our patients had markedly aperiodic vibratory cycles at the time of diagnosis, but a statistically significant improvement in periodicity values was obtained after surgery, which indicated that our patients regained successive vocal vibratory cycles.

All of the above-mentioned reasons causing vibratory anomalies and glottic closure defects might also lead to supraglottic contraction with hyperfunction. These contrac- tions might occur in antero-posterior and/or latero-medial directions and prevent vocal fold visualisation. In our patients, antero-posterior contraction values were significantly decreased after surgery. This decrease might be attributed to the success of our surgical method in eliminating hyperfunctional behaviours that develop as a consequence of glottic pathology.

In the present study, false cord vibration was the only parameter that did not improve significantly after surgery. False cord vibrations develop as a compensatory mechanism to the long-term vibratory distortion at the glottic region (7). Similarly, our patients had significantly higher scores before surgery than control subjects with regard to false cord vibration parameters. Unfortunately, their scores did not decrease enough after surgery. We believe that this is because 6 months is not a sufficient postoperative time to improve this compensatory alteration. We assume that we can also achieve significant improvements in this parameter later, but studies that analyse the postoperative period after 6 months and have a longer follow-up time are warranted to evaluate that implication.

In conclusion, surgical excision is a primary treatment modality for vocal fold hyperplastic epithelial lesions. The hydrodissection technique applied under high quality operating microscopes with appropriate microsurgical instruments may be beneficial for safe excision. VLS supported by suitable software is a valuable examination method in the follow-up of recurrence and functional consequences of surgery.

Ethics Committee Approval: Ethics committee approval was received for this study from the ethics committee of Eskişehir Osmangazi University Faculty of Medicine (reference number: 2009-28).

Informed Consent: Written informed consent was obtained from each patient and from the participants of control group.

Peer-review: Externally peer-reviewed.

Author contributions: Concept - E.Ö., M.K.G.; Design - E.Ö., M.K.G.; Supervision - E.Ö., C.C., A.I.; Resource - E.Ö., M.K.G., M.F.A.; Materials - E.Ö., M.K.G., M.F.A.; Data Collection\&/or Processing - M.K.G., L.B.; Analysis\&/or Interpretation - M.F.A., E.Ç.; Literature Search - M.K.G., L.B.; Writing - M.K.G.; Critical Reviews - E.Ö., C.C., A.i.

Conflict of Interest: No conflict of interest was declared by the authors. Financial Disclosure: No financial disclosure was declared by the authors.

\section{References}

1. Blackwell KE, Calcaterra TC, Fu YS. Laryngeal dysplasia epidemiology and treatment outcome. Ann Otol Rhinol Laryngol 1995;104:596-602.

2. Gugatschka M, Kiesler K, Beham A, Rechenmacher J, Friedrich G. Hyperplastic epithelial lesions of the vocal folds: combined use of exfoliative cytology and larygostroboscopy in differential diagnosis. Eur Arch Otorhinolaryngol 2008;265: 797-801. [CrossRef]

3. Hussein MR. Alterations of $\mathrm{p} 53$ and $\mathrm{Bcl}-2$ protein expression in the laryngeal intraepithelial neoplasia. Cancer Biol Ther 2005;4:213-7. [CrossRef]

4. Gallo A, de Vincentiis M, Della Rocca C, Moi R, Simonelli M, Minni A, et al. Evolution of precancerous larygeal lesions: A clini- 
copathologic study with long- term follow-up on 259 patients. Head Neck 2001;23:42-7. [CrossRef]

5. Sadri M, McMahon J, Parker A. Management of larygeal dysplasia: a review. Eur Arch Otorhinolaryngol 2006;263:843-52. [CrossRef]

6. Remacle M, Eckel HE, Antonelli A, Brasnu D, Chevalier D, Friedrich $\mathrm{G}$, et al. Endoscopic cordectomy. A proposal for a classification by the Working Committee, European Laryngological Society. Eur Arch Otorhinolaryngol 2000;257:227-31. [CrossRef]

7. Hirano M, Bless DM. Videolaryngostroboscopic examination of the larynx. Singular Publishing Group, San Diego, 1993.
8. Anghelina $F$, loniţă $E$, Popescu CF, loniţă I, Mogoantă C, Ciolofan $S$, et al. Clinical, morphological and immunohistochemical aspects in laryngeal premalignant lesions. Rom J Morphol Embryol2006;47:169-74.

9. Fiorella R, Di Nicola V, Resta L. Epidemiological and clinical relief on hyperplastic lesions of the larynx. Acta Otolaryngol Suppl 1997;527:77-81. [CrossRef]

10. Gamboa J, Echeverría L, Molina B, Cobeta I. Stroboscopic assessment of chronic laryngitis. Acta Otorhinolaryngol Esp 2006;57:266-9. [CrossRef] 